\title{
The Materials Science Community and the National Synchrotron Light Source
}

\begin{abstract}
"Our understanding of materials properties and phenomena can only be as sophisticated as the techniques we can use to probe them." This is one of the most used and abused sentences in modern scientific vernacular. Directors of research or funding programs wince when they hear it because they know what comes next.

But it's true! Frontier materials science research is concerned with increasingly elegant and subtle effects, and if one day this materials science is to become materials technology we must have the tools to match the problem.
\end{abstract}

There has been a virtual revolution in the capabilities of modern analytical techniques. But many of these modern analytical techniques are becoming prohibitively expensive and require considerable expertise and resources to operate and main tain. Furthermore, the nature of modern materials problems dictates that one must have access to multiple techniques in order to fully span the parameter space necessary for new insight. Thus the individual scientist more and more of ten seeks out the latest capability which is complementary to his own and collaborates in interdisciplinary and interinstitutional ways in order to make progress.

The role of our national materials research facilities is to provide for the general community capabilities which no single researcher or group of researchers could afford to acquire or maintain, but which are indispensable to the science. This mode of operation has long been the norm in high energy physics, astronomy, space explora- tion, and plasma physics-and it has led to great gains.

The potential of synchrotron radiation as the basis for sophisticated analytical techniques to study the properties and phenomena connected with today's advanced materials has been understood for almost two decades. During this period, scientists from many disciplines-ranging from solid-state physics through metallurgy, life science, and geoscience-have been drawn to the area and have developed experimental techniques and methodologies which have opened new vistas in their science and lent a new vitality to the entire field of materials science.

Early successes led to planning in the mid 70 s for an extensive expansion in capability and capacity to make synchrotron radiation available to a larger community and to provide instrumentation and sufficient beamtime so that new classes of experiments and a wider community could be accommodated. The National Synchrotron Light Source (NSLS) at Brookhaven National Laboratory, which came out of this initiative, was built by the U.S. Department of Energy to provide synchrotron radiation to the widest possible community in support of its mission of providing for a strong materials science effort for energy, defense, and economic vitality-not only within its own programmatic interests, but across the full range of national interests and funding agencies.

At the NSLS, the long materials sciences tradition of distributing the intellectual and resource base among diverse small research groups has been preserved by utilizing the concept of "participating research teams." Consortia of researchers from industry, academia, and government laboratories have pooled their resources and research goals to put together beamlines and experimental stations tailored to their needs. They receive $75 \%$ of the beamtime for their own experimental programs and provide $25 \%$ of the time to the NSLS to distribute to the "general user" who applies for beamtime on a proposal basis. Both groups receive their experimental time at no charge unless they perform proprietary research. The materials scientist can become involved at any level-from the development of new capability with significant emphasis on its use to the occasional measurement to complement other work.

Within the past year, the plans of the last decade have come to fruition and the capacity for synch rotron radiation research has increased by an order of magnitude in the United States. The NSLS is joined by an expanded Stanford Synchrotron Radiation Laboratory, the new $1 \mathrm{GeV}$ ring at the Synchrotron Radiation Center at the University of Wisconsin, CHESS at Cornell University, and SURF at the National Bureau of Standards. It is important that the materials science community realize that the capability is here, that it is for their use, and that it is available for the asking.

M.L. KNOTEK, Chairman National Synchrotron Light Source Brookhaven National Laboratory

\section{Do You Have An Opinion?}

\author{
The MRS BULLETIN wants your comments \\ and views on issues affecting materials research. \\ Send your comments to: \\ Editor \\ MRS BULLETIN \\ 9800 McKnight Road, Suite 327 \\ Pittsburgh, PA 15237 \\ Telephone: (412) 367-3036
}




\section{Another New ldea}

\section{Reactive and Inert Gas Atom/lon Milling}

The FAB306B now provides milling with both reactive and inert gases. The Saddle Field Source based Atom/lon Mill' provides collimated atom or ion beams for stable milling periods of 250 hours without cathode erosion or beam spread. Termination by collecting a pulsed, transmitted ion beam through the specimen insures precise and repeatable end point conditions with all types of specimens including those that become optically transparent when thin. The constant and high milling rates of the SF Sources eliminate gas fluctuation problems and the need for two milling chambers.

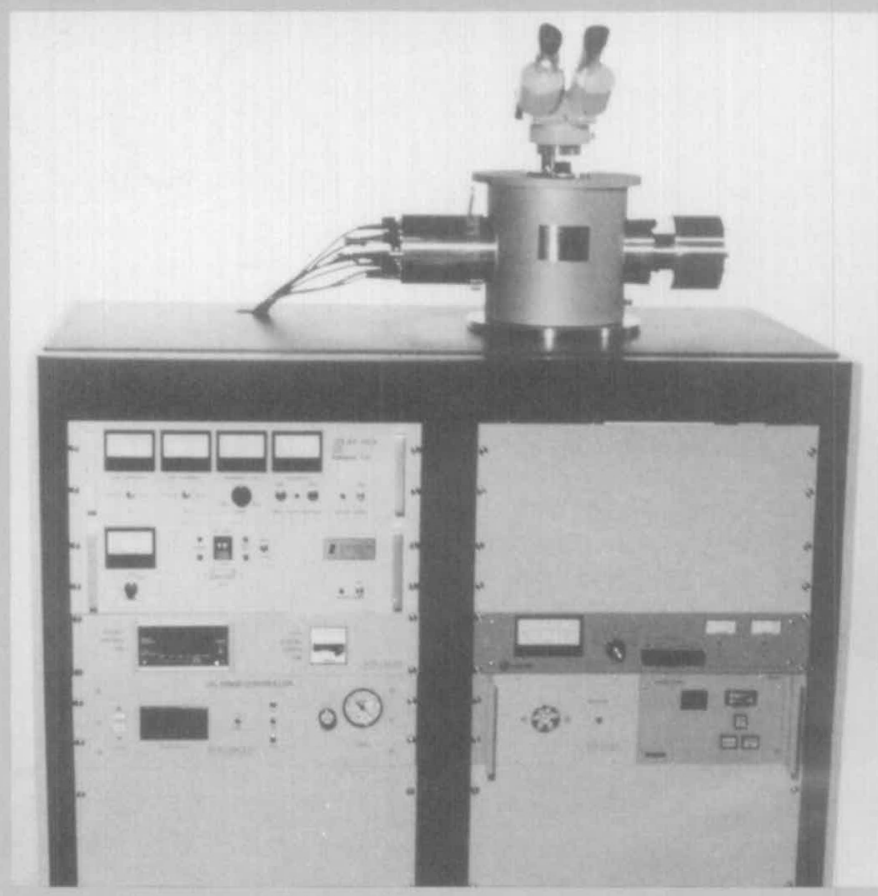

\section{DIMPLER" Model D500}

The Dimpler, introduced by VCR Group in 1982, now incorporates a microprocessor based LED displayed Preset/ Elapsed Timer. Also, Translucent Specimen Platens illuminated from below allow specimen viewing while in the dimpling position as desired final thickness is approached
The guaranteed tolerance between tools and specimen surface is $<3.8 \mu \mathrm{m}$.

\section{Services}

Dimpling and milling services for difficult or unique specimen materials available.

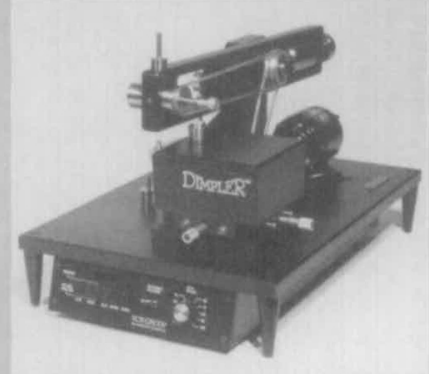

\section{Gryotrans System GT1000A2}

The Cryotrans system allows viewing frozen hydrated samples in the SEM without condensation artifacts. Developed by Hexland's electron microscopist, the CT1000A utilizes a preparation chamber that is integral to the SEM. This allows DC or ion beam sputter coating, evaporative coating and controlled cutting or honing for $\mathrm{X}$-ray analysis. Easy placement onto the SEM cold stage for repeated observations is facilitated by a lamp and viewing window.

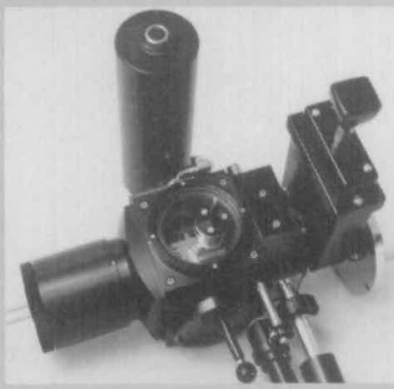

\section{Also New ...}

- Heating Stages ${ }^{2}$ for SEM with temperatures to $1500^{\circ} \mathrm{C}$ Cold Stages ${ }^{2}$ for SEM with microprocessor controlled temperatures from $-185^{\circ}$ to $400^{\circ} \mathrm{C}$.

- Tensile Stages ${ }^{2}$ for SEM up to $80 \mathrm{~kg}$ for textiles and fibers.

Heating Holder and

Environmental Cell for TEM ${ }^{2}$

\section{Services}

$X$-ray Fluorescence Services: Elements from sodium to uranium provide uniform sensitivity not achievable with EDS. Applications include wear metals from lube oils, trace element analysis in liquids, precious metals and more. 